\title{
Focal cryoballoon versus radiofrequency ablation of dysplastic Barrett's esophagus: impact on treatment response and postprocedural pain
}

Sanne N. van Munster, MD ${ }^{1,2}$ Anouk Overwater, MD,${ }^{1,3}$ Rehan Haidry, MD, PhD, ${ }^{4}$ Raf Bisschops, $\mathrm{MD}, \mathrm{PhD},{ }^{5}$ Jacques J. G. H. M. Bergman, MD, ${ }^{2}$ Bas L. A. M. Weusten, $\mathrm{MD}^{1,3}$

Nieuwegein, Amsterdam, Utrecht, the Netherlands; London, United Kingdom; Leuven, Belgium

GRAPHICAL ABSTRACT

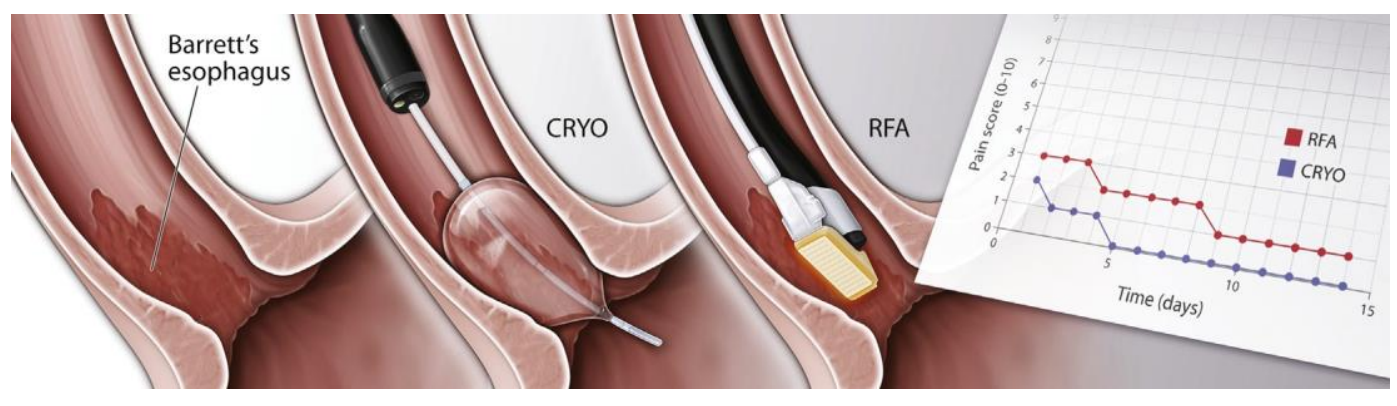

Background and Aims: Radiofrequency ablation (RFA) is safe and effective for eradicating Barrett's esophagus (BE) but is associated with significant postprocedural pain. Alternatively, balloon-based focal cryoablation (CRYO) has recently been developed, which preserves the extracellular matrix and might therefore be less painful. Although data for CRYO are still limited, uncontrolled studies suggest comparable safety and efficacy to RFA in eradicating limited $\mathrm{BE}$ areas. Therefore, secondary endpoints such as pain might become decisive for treatment selection. We aimed to compare efficacy and tolerability between focal CRYO and RFA.

Methods: We identified BE patients undergoing focal ablation (either RFA or CRYO) of all visible BE from our prospective cohort in 2 Dutch referral centers. After ablation, patients completed a 14-day digital diary to assess chest pain (0-10), dysphagia (0-4), and analgesics use. A follow-up endoscopy was scheduled after 3 months to assess the $\mathrm{BE}$ surface regression (blindly scored by 2 independent BE expert endoscopists). Outcomes were BE surface regression; 14-day cumulative scores (area under the curves [AUCs]) for pain, dysphagia, analgesics, and peak pain.

Results: We identified 46 patients (20 CRYO, $26 \mathrm{RFA}$ ) with similar baseline characteristics. The BE regression was comparable ( $88 \%$ vs $90 \%, P Z$.62). AUCs for pain, dysphagia, and analgesics were significantly smaller after CRYO versus RFA (all $P<.01)$. Peak pain was lower after CRYO (visual analog scale 2 vs $4, P<.01$ ), and the duration of pain was also shorter after CRYO ( 2 vs 4 days, $P<.01$ ). CRYO patients used analgesics for 2 days versus 4 days for RFA $(P<.01)$.

Conclusions: In this multicenter, nonrandomized cohort study, we found no differences in efficacy after a single treatment with CRYO and RFA for short-segment BE. Patients reported less pain after CRYO as compared with RFA. Moreover, CRYO patients used fewer analgesics. Our results suggest a different pain course favoring CRYO over RFA, but a randomized trial is needed for definitive conclusions. (Clinical trial registration number: NCT02249975.) (Gastrointest Endosc 2018;88:795-803.)

The incidence of esophageal adenocarcinoma (EAC) has increased 6-fold in the last 3 decades, making it the most rapidly increasing cancer in the Western world. ${ }^{1}$ EAC arises from Barrett's esophagus (BE), which is defined as intestinal metaplasia of the esophageal epithelium. ${ }^{2,3}$ As metaplastic cells develop progressive cellular atypia, classi- fied as dysplastic BE, the risk for development of EAC increases significantly. ${ }^{4}$ Therefore, dysplastic BE is an indication for endoscopic treatment. ${ }^{5,6}$

Radiofrequency ablation (RFA) is currently the most widely used endoscopic ablation therapy for 
eradication of flat-type BE. In a wide range of clinical studies including several randomized trials, this heatbased ablation method has been proven to be effective in eradication of $\mathrm{BE}$ with an acceptable safety profile.7-12 Although it is not systemat- ically studied, it is clinically recognized that patients often report substantial postprocedural pain after RFA.13

Endoscopic cryoballoon ablation is a relatively new tech- nique that ablates BE through intracellular ice formation, cell rupture, and hypoxia.14,15 In contrast to heat-based ablation, cryoablation preserves the extracellular matrix and tissue architecture.14 Studies investigating the efficacy and safety of cryoablation using the cryoballoon focal ablation system (CbFAS) have shown the feasibility of $\mathrm{BE}$ eradication in up to $100 \%$ of small $\mathrm{BE}$ islands, with acceptable safety profiles.16-18 When compared with RFA, improved patient tolerance may constitute one of the ad- vantages of cryoablation, but to date no data support this. Potential underlying mechanisms previously described include direct effects of cooling, including an anesthetic effect and blocked nerve conduction; delayed mucosal injury; and/or preservation of the tissue architecture.15,19-21 If efficacy and safety are comparable, postprocedural side effects like patient tolerance may be of importance in select- ing the preferred ablation tool.

To date, no trials have compared CbFAS with RFA. We aimed to systematically compare endoscopic $\mathrm{BE}$ ablation using $\mathrm{CbFAS}$ and focal RFA with respect to eradication rates and postprocedural pain. We hypothesize that CbFAS is less painful compared with RFA, with comparable efficacy.

\section{METHODS}

Study setting and patient selection

We performed a retrospective analysis of prospectively collected data. Data were derived from the postprocedural pain after endoscopic therapy for BE (P-PET) cohort. This cohort consisted of patients who completed an electronic diary assessing postprocedural pain and dysphagia after endoscopic therapy for BE in 3 expert centers in the Netherlands (St Antonius Hospital [Nieuwegein], Aca- demic Medical Center [Amsterdam], and University Medi- cal Center [Utrecht]). The diary consisted of daily surveys through 14 days and was initially used for clinical purposes. 
All data were prospectively and anonymously gathered in the P-PET cohort. All patients consented to use of their data in the P-PET cohort for the purpose of research. The need for ethics approval was waived by the Medical Research Committees United.

We retrospectively reviewed the P-PET cohort and included all patients with flat BE and an indication for focal ablation therapy, either confirmed low-grade dysplasia (LGD), confirmed high-grade dysplasia, residual $\mathrm{BE}$ after endoscopic resection for nonflat lesions containing any degree of dysplasia or lowrisk mucosal EAC (ie, not poorly differentiated, negative deep resection margins, and absence of lymphatic and vascular invasion), or residual $\mathrm{BE}$ after circumferential or focal ablation performed for one of the indications listed above. Other inclusion criteria were completion of the electronic diary after endoscopic therapy with either CbFAS (CRYO group) or focal RFA (RFA group) and at least 1 follow-up endoscopy. An exclusion criterion was the presence of a stenosis before treatment. If patients completed the electronic diary multi- ple times (ie, if multiple focal ablation sessions were per- formed), we only included the surveys after the first treatment to prevent duplications and/or repeated measurements.

\section{Endoscopic procedures}

Both CbFAS and RFA procedures were performed on an outpatient basis, with the patient under conscious sedation with midazolam and alfentanil or monitored anesthesia us- ing propofol. Both procedures were performed by endo- scopists highly experienced in endoscopic treatment of $\mathrm{BE}$ (B.L.A.M.W. and J.J.G.H.M.B.).

High-resolution white-light endoscopy and narrow-band imaging were used for all procedures. The $\mathrm{BE}$ segment was carefully inspected, the Prague $\mathrm{C} \& \mathrm{M}$ criteria were docu- mented with addition of the location of the most proximal islands, 22 and still images of the entire BE segment were acquired. All patients underwent ablation of all visible $\mathrm{BE}$ in addition to a circumferential ablation of the esophagogastric junction. After the procedure, all patients were advised in a standardized way to use oral paracetamol (maximum $1000 \mathrm{mg} 3$ times a day) in case of pain, with additional rectal diclofenac (maximum $100 \mathrm{mg}$ twice a day) if needed. We guaranteed low threshold and easy contact with the clinical team. All patients received high-dose proton pump inhibitors after treatment (equivalent of esomeprazole $40 \mathrm{mg}$ twice daily). In addi- tion, patients were prescribed ranitidine $300 \mathrm{mg}$ before bed and $5 \mathrm{~mL}$ sucralfate suspension 3 times daily for a period of 2 weeks after the procedure, as is the standard of care in the Netherlands.

\section{CRYO group}

Cryoablation was performed using CbFAS (C2 Thera- peutics, Inc, Redwood City, Calif). A precise description of this therapy has been outlined in earlier studies 16,17

The system comprises a handheld, through-the-scope system with a conformable balloon that is simultaneously inflated and cooled using nitrous oxide, resulting in ice patches of approximately 2 $\mathrm{cm} 2$ on the targeted mucosa. All patients were treated with side-by-side applications of 10 -seconds duration per application.16,23 All CRYO treat- ments were performed in context of a feasibility study registered at clinicaltrials.gov with number NCT02249975.

\section{Focal RFA group}

Focal RFA (Medtronic, Inc, Minneapolis, Minn) has been described earlier.9,24,25 The technique uses a bipolar electrode that can be mounted as a cap on the tip of the endoscope. Pa- tients were treated with either a focal RFA regimen consisting of 3 applications with $12 \mathrm{~J} / \mathrm{cm} 2$ or a regimen that consisted of 2 applications with $12 \mathrm{~J} / \mathrm{cm} 2$ followed by a cleaning step and another 2 applications with $12 \mathrm{~J} / \mathrm{cm} 2$.

\section{Electronic diary}

All patients completed an electronic diary through 14 days after treatment to report pain and dysphagia scores and use of pain medication (Supplementary Fig. 1, available online at www.giejournal.org). Patients were asked to rate retrosternal pain in rest (question 1) and during eating and drinking (question 2), both on a numeric rating scale ranging from 0 (no pain) to 10 (unbearable pain). We used a validated score to assess dysphagia ranging from 0 to 4 (question 3).26 Last, patients were asked whether they had used pain medication in the last 24 hours (question 4). If patients had 
missing data in the electronic diary, we attempted to add information derived from research-related phone calls. We only added data if all the following items were explicitly mentioned: date and time, pain score (range, 0-10), dysphagia score (range, 0-4), and use of pain medication. Verbal pain scores have been shown to correlate well with numeric rating scale pain scores. 27

Efficacy assessment

A follow-up endoscopy was petrformed 31 months postprocedure to thoroughly inspect the esophagus for the presence of residual $\mathrm{BE}$, stenosis, and other abnormal- ities. One still image was made on every $1 \mathrm{~cm}$ of the original $\mathrm{BE}$ segment. The $\mathrm{BE}$ surface regression was defined as the percentage of initial $\mathrm{BE}$ that had been con- verted to squamous epithelium. This was assessed by review of endoscopic images of the $\mathrm{BE}$ segment captured immediately before the initial ablation and during follow- up endoscopy. This was blindly and independently scored in randomized order by 2 endoscopists (R.H. and R.B.), both highly experienced in BE-related endoscopies, both working in centers other than the study hospitals, and both uninvolved in treatments or decision-making for the study patients. In the event the percentage differed by $30 \%$ or more, a meeting was held to establish a consensus score. The endoscopists indicated whether the endoscopic images were representative with regard to quality and quantity, scored as excellent, good, fair, or poor.

\section{Histopathologic analysis}

In all CRYO patients, in context of the previously mentioned feasibility study, 4-quadrant biopsy specimens were taken just below the neosquamocolumnar junction and for every $2 \mathrm{~cm}$ of the original $\mathrm{BE}$ segment (either from neosquamous epithelium or residual $\mathrm{BE}$ ). In the RFA patients no biopsy specimens were taken in line with regular clinical care, because robust data have shown that endoscopically visible neosquamous epithelium after RFA treatment will be confirmed histopathologically.7,28

Biopsy samples were fixed in formalin (10\%), embedded in paraffin, and stained with hematoxylin and eosin. All specimens, including baseline endoscopic resection speci- mens, baseline biopsy samples, and all biopsy specimens taken thereafter were examined by an expert pathologist in the field of GI pathology (S. Meijer or K. Seldenrijk).

\section{Primary outcomes}

We defined 2 primary endpoints for this study. The first primary endpoint was efficacy, defined as the $\mathrm{BE}$ surface regression after a single focal ablation treatment. The sec- ond primary endpoint was cumulative pain through 14 days. Cumulative pain is a composite score based on the 2 pain-related questions in the electronic diary (questions 1 and 2) over the entire 14-day period. The daily pain scores, defined as the maximum pain score of questions

1 and 2 per day, were depicted in a daily pain versus time plot through 14 days for each patient. Cumulative pain was defined as the area under the pain curve (AUC) for this pain intensity versus time plot.

\section{Secondary outcome parameters}

Several secondary outcomes were reported: (1) the duration of pain, defined as the number of days until daily pain scores were reported to be 0 on all following days, and (2) the duration of major pain as time until daily pain scores were 3 or less. Peak pain score (3) was the maximum pain score reported on any of the 14 days. Cumulative use of pain medication (4) was defined as the AUC of a pain medication (question 4) versus time plot. Cumulative dysphagia (5) was assessed by the AUC of a dysphagia (question 3) versus time plot.

\section{Statistics}

Data analysis was performed using IBM SPSS statistical software package (SPSS Inc, Chicago, Ill). Means with stan- dard deviations were computed for normally distributed variables and medians with interquartile ranges (IQRs) for variables with a skewed distribution. Categorical vari- ables were presented as frequencies and percentages of total. Continuous variables with a normal or skewed distri- bution were compared using the Student $t$ test or Mann- Whitney $U$ test, respectively. Categorical variables were compared with the $\mathrm{c} 2$ test or the Fisher exact test. The duration of pain 
and pain medication was assessed with the Kaplan-Meier analysis, and the 2 groups were compared with a log rank test.

We performed sensitivity analysis to test the consistency and robustness of our findings. An additional analysis with adjustment for potential confounders (ie, age, gender, BE length, and prior treatment) was performed using a multi- variable linear regression model.

\section{RESULTS}

\section{Subjects}

We identified 46 patients from the P-PET cohort that were treated between April 2016 and February 2017; 20 were treated with CbFAS (CRYO group) and 26 with focal RFA (RFA group) (Figs. 1 and 2). The proportions of patients who previously underwent both endoscopic resection and ablation, endoscopic resection only, or ablation therapy only were $20 \%, 30 \%$, and $20 \%$, respectively, in the CRYO group and $27 \%, 19 \%$, and $34 \%$, respectively, in the RFA group ( $P$ Z .54). The mean BE length before the current focal treatment was COM2 in the CRYO group and COM 1 in the RFA group $(P>.25)$. There were no significant differences with regard to any other baseline variable for the 2 groups (Table 1). In total, 558 of 644 expected daily surveys (87\%) were completed and $86(13 \%)$ were missing. Thirty-one patients $(67 \%)$ completed all 14 daily surveys, whereas 15 patients $(33 \%)$ missed 1 or more surveys. 


\section{Efficacy}

There were no statistically significant differences for the BE surface regression at 3 months followup between the 2 groups: $88 \%$ (IQR, 63-94) for CRYO and $90 \%$ (IQR,

77-94) for RFA ( $P$ Z .62) (Fig. 3). A median of 9 (IQR, 7-11) endoscopic images were available per patient. The median difference in BE surface regression between the 2 assessors was $10 \%$ (IQR, 5-20) and for 2 patients with a difference $>30 \%$, the percentage was established in a consensus meeting. The images of 3 patients were scored as poor for either quantity or quality (Table 2). For 2 of these patients, no BE surface regression could be determined, and therefore these patients were excluded from efficacy analysis. In the CRYO patients a total of 87 biopsy samples were taken from endoscopically eradicated areas, neosquamous epithelium was confirmed histologically in all 87 biopsy samples, and no subsquamous BE glands were found. In

17 patients with residual $\mathrm{BE}$, a total of 43 additional biopsy samples were taken from the residual BE epithelium, with pathology assessment showing squamous epithelium in $7(16 \%)$, nondysplastic BE in 28 (65\%), and BE with LGD in $8(19 \%)$. The 8 biopsy specimens containing LGD were derived from 3 patients with a baseline diagnosis of LGD (nZ2) or EAC (n Z1).

\section{Cumulative pain}

Cumulative pain through 14 days was significantly less in the CRYO group compared with the RFA group (Fig. 4). Median AUCs for pain intensity versus time plots were 4 (IQR, 0-16) for the CRYO group and 22 (IQR, 14-44) for the RFA group $(P<.01)$. Median pain scores at each single day were consistently lower in the CRYO group (Fig. 4). Sensitivity analysis adjusting for age, gender, BE length, and prior treatment showed that CRYO was associated with a significantly smaller AUC $(P<.01$; Supplementary Table 1 , available online at www.giejournal.org). 


\section{Secondary outcomes}

\pm The duration of pain was significantly shorter for the CRYO group compared with the RFA group (Fig. 4). Patients in the CRYO group were free of pain after 5.7 1.1 days, compared with 11.1 1.0 days for the RFA group $(P<.01)$. The duration of major pain was similarly shorter for the CRYO grouptwhen compared with the RFA group ( $3.5 \pm .9$ and 6.51 .0 days, respectivety; $P Z$.04). Peak pain score was significantly lower after CRYO compared with RFA (median visual analog scale 2 [IQR, 0-4] vs 4 [IQR, 3$7], P<.01)$. CRYO patients reported peak pain a median of 2 days (IQR, 1-2) after treatment, compared with 1 day (IQR, 1-4) for the RFA group (PZ .95).

CRYO patients used significantly less analgesics compared with RFA patients (Fig. 4). Patients in the CRYO group used pain medication for 2.6 .7 days compared with $6.3 \quad 1.0$ days in the RFA group $(P<$

$\pm$

$.01)$. In th $\notin$ CRYO group, $2(10 \%)$ patients used paracetamol and $3(15 \%)$ used nonsteroidal anti- inflammatory drugs, whereas this was $15(58 \%)$ and $3(12 \%)$ patients, respectively, in the RFA group (PZ .09). CRYO patients reported significantly less dysphagia compared with RFA patients. No patients in the CRYO group and 2 patients $(8 \%)$ in the RFA group developed an esophageal stenosis after treatment. No other adverse events were reported in either of the 2 groups.

\section{DISCUSSION}

This multicenter study of prospectively collected data shows that although the efficacy after a single treatment with CRYO and RFA for eradication of short-segment BE is comparable, CRYO is associated with less postprocedural pain and dysphagia than focal RFA. Moreover, CRYO patients used less analgesics, and this use was of shorter duration. Although a randomized trial is needed for defin- itive conclusions, our data suggest differences in postpro- cedural tolerability favoring CRYO over RFA.

RFA is a heat-based ablation technique and can be applied using circumferential or focal devices. Multiple large-scale studies of RFA have shown robust and repro- ducible data on efficacy and safety outcomes, and RFA is therefore recommended as the standard ablation tech- nique in current clinical guidelines. ${ }^{5,29}$

CRYO is a relatively new ablation method, which causes cell death through freezing. Application of liquid nitrous oxide cools the mucosa to approximately $-850 \mathrm{C}$ and results in mucosal ablation. CbFAS is currently the most commonly used device for CRYO, but data on efficacy, safety, and durability are limited and preliminary.

The first important criterion in selecting the preferred treatment is efficacy. Our study demonstrated no statistically significant differences in BE surface regression rates after CbFAS and focal RFA for eradication of short-segment BE. The efficacy rates we found for CRYO and RFA are in line with previous studies, showing regression rates of $82 \%$ to $100 \%$ and $78 \%$ to $90 \%$ after a single treatment with CbFAS (for small BE areas only) and RFA, respectively. 16,17,30,31 When assessing the proportion of patients achieving a com- plete eradication of $\mathrm{BE}$ on consecutive treatment sessions, rates vary between $77 \%$ and $93 \%$ after RFA,10,12,32 and the only study on CbFAS reported a 1-year success proportion of $88 \%$ for complete eradication of intestinal metaplasia.33 The efficacy results of the current study can be considered an early and rough estimation, and we therefore encourage additional prospective studies on CbFAS alone as well as in comparison with RFA to further assess the role of CbFAS in treatment of BE.

Robust data have already shown that endoscopically eradicated areas after RFA will contain neosquamous epithelium without subsquamous BE glands in histol- ogy.7,28 Because these data are lacking for CRYO, we per- formed additional biopsy sampling from endoscopically eradicated areas after CbFAS. All these biopsy specimens from endoscopically eradicated areas were confirmed histologically to contain neosquamous epithelium with no evidence for subsquamous BE glands.

This is the first study that systematically compared patient tolerability after CRYO and RFA, and this is relevant because improved patient tolerability might constitute one of the most important advantages of CRYO over RFA. We found significantly lower pain and dysphagia scores after CRYO, and, moreover, CRYO patients used significantly less analgesics.

The exact mechanism for the differences in pain remains unknown, but several factors may play a role. Cryoablation might reduce pain through a direct anesthetic effect of cooling the mucosa and its surrounding, 15 and pain transmission might be minimized because cold temperature decreases or blocks nerve conduction. 19 In addition to electrophysiologic effects, vasoconstriction of blood vessels 
may minimize edema and reduce the release of pain-producing substances from damaged tissue.34 Others speculate that the delay in mucosal injury after cryoablation might play a role. 20 Cryoablation leaves the tissue architecture intact, whereas RFA results in denaturation of proteins and permanent changes in tissue structure.21 It is interesting to note that the choice between CRYO and RFA also applies to other fields of medicine, such as cardiac ablation for atrial flutters or ablation of solid tumors in liver, kidney, or bone. A large number of comparative studies including several randomized trials in this regard consistently demonstrate that CRYO is less painful compared with RFA. ${ }^{19,35-37}$

Although no concrete definition exists for clinically rele- vant differences in pain, a difference of $20 \%$ is frequently reported in this regard.38,39 Both the cumulative pain score (median 22 vs 4) and the peak pain score (median 4 vs 2 ) after RFA were $>20 \%$ higher compared with CRYO. The absolute pain scores were, however, relatively low.

Because this may reflect the limited size of $\mathrm{BE}$, and thus the limited extent of ablation, in our study population, comparisons in larger BE areas will be of interest. The dif- ferences in use of pain medication can also be considered an indication of clinical relevance. In summary, we believe that our results indicate clinically relevant differences in pain between CRYO and RFA, and we encourage compar- isons for larger BE areas.

Our study has several strengths. All data were prospec- tively collected. All patients were treated in a homoge- neous fashion, consisting of circumferential treatment of the gastroesophageal junction and ablation of all endo- scopically visible BE. We quantified the complete course of postprocedural pain and dysphagia through 14 days after treatment, and we specifically asked the patients to score retrosternal pain to filter out other causes of pain. The response rate in our study was high: All patients completed the day- 1 survey and all but 9 completed the day-14 survey; however, all 9 patients reported no pain or dysphagia at their last assessment. The influence of missing surveys in between was further minimized by the assessment of AUCs. The BE surface regression percentage was independently and blindly assessed in random order by $2 \mathrm{BE}$ expert endoscopists.

Our study has several limitations as well, including 3 major limitations. First is the nonrandomized design of our study with 2 important implications. Although baseline characteristics were not significantly different between the 2 groups and additional analysis that was corrected for potential confounders found similar results, other unknown confounding factors might have influenced our findings. We have no data on the baseline pain score, and the median pain score in the RFA group is still $>0$ after 14 days. This can be caused either by the severity of pain after RFA or by other pre-existing differences (ie, in patient characteristics and/or baseline pain scores). Second, patients were not blinded for treatment. All CRYO treat- ments were performed in the context of a clinical trial, whereas RFA treatments were not, and psychological effects in this regard might have influenced the pain scores. We tried to minimize this by informing patients in an identical way on pain and analgesics.

The second major limitation is the limited length of the BE segment in our study population. Longer $\mathrm{BE}$ length is known to be associated with increased postprocedural pain and decreased efficacy. Therefore, our conclusions are applicable to focal ablation treatment for patients with short-segment BE $(<3 \mathrm{~cm})$, and the generalizability to treat- ment of longer BE segments should be cautioned against.

The third major limitation applies to the efficacy endpoint, which was assessed as a derivate endpoint after a single treatment session. Because current clinical guide- lines advocate consecutive treatment sessions until all BE is eradicated, this is an important field for further research. Moreover, we included patients with relatively short-segment $\mathrm{BE}$ in absence of a stenosis, and prior 
endoscopic therapy was allowed. Thereby we might have selected those patients with a high chance for a suc- cessful outcome.

Other limitations include a lack of a pain survey directly after the procedure and a clinically relevant question, such as return to normal daily activities. We, however, decided to start the diary not directly post-treatment given the vari- ation in intravenous analgesic administration during endo- scopic ablation and to minimize the number of questions. The type of sedation was not standardized; however, given the relatively short-term effects of both propofol and mida- zolam, the effects on the postprocedural pain course from day 1 to day 14 would be negligible. The multivariable anal- ysis included too many variables in relation to the number of patients included in this study, with a risk for overfitting. Some of the secondary outcomes might relate to each other, like the peak pain score and the duration of pain.

In conclusion, this study shows that a single treatment with CRYO might be comparably effective with focal RFA for treatment of short-segment BE. We also show that CRYO is better tolerated in terms of pain and dysphagia as compared with RFA in patients with short-segment BE. We encourage validation of our findings in a randomized trial.

\section{ACKNOWLEDGMENTS}

We would like to thank Sybren Meijer and Kees Selden- rijk, expert pathologists for Barrett's esophagus, for their contribution to this study.

\section{REFERENCES}

1. Pohl H, Welch HG. The role of overdiagnosis and reclassification in the marked increase of esophageal adenocarcinoma incidence. J Natl Can- cer Inst 2005:97:142-6.

2. Drewitz DJ, Sampliner RE, Garewal HS. The incidence of adenocarci- noma in Barrett's esophagus: a prospective study of 170 patients fol- lowed 4.8 years. Am J Gastroenterol 1997;92:212-5.

3. O'Connor JB, Falk GW, Richter JE. The incidence of adenocarcinoma and dysplasia in Barrett's esophagus: report on the Cleveland Clinic Barrett's Esophagus Registry. Am J Gastroenterol 1999:94:2037-42.

4. Rastogi A, Puli S, El-Serag HB, etal. Incidence of esophageal adenocar- cinoma in patients with Barrett's esophagus and high-grade dysplasia: a meta-analysis. Gastrointest Endosc 2008;67:394-8.

5. Weusten B, Bisschops R, Coron E, etal. Endoscopic management of Barrett's esophagus: European Society of Gastrointestinal Endoscopy (ESGE) position statement. Endoscopy 2017;49:191-8.

6. ASGE Standards of Practice Committee; Evans JA, Early DS, Fukami N, et al. The role of endoscopy in Barrett's esophagus and other prema- lignant conditions of the esophagus. Gastrointest Endosc 2012;76: 1087-94.

7. Phoa KN, Pouw RE, van Vilsteren FG, etal. Remission of Barrett's esoph- agus with early neoplasia 5 years after radiofrequency ablation with endoscopic resection: a Netherlands cohort study. Gastroenterology 2013;145:96-104.

8. van Vilsteren FG, Pouw RE, Seewald S, et al. Stepwise radical endo- scopic resection versus radiofrequency ablation for Barrett's oesoph- agus with high-grade dysplasia or early cancer: a multicentre randomised trial. Gut 2011;60:765-73

9. Pouw RE, Wirths K, Eisendrath $\mathrm{P}$, et al. Efficacy of radiofrequency abla- tion combined with endoscopic resection for Barrett's esophagus with early neoplasia. Clin Gastroenterol Hepatol 2010;8:23-9.

10. Shaheen NJ, Sharma P, Overholt BF, et al. Radiofrequency ablation in Barrett's esophagus with dysplasia. N Engl J Med 2009;360:2277-88.

11. Fleischer DE, Overholt BF, Sharma VK, et al. Endoscopic radiofrequency ablation for Barrett's esophagus: 5-year outcomes from a prospective multicenter trial. Endoscopy 2010;42:781-9.

12. Phoa KN, van Vilsteren FG, Weusten BL, et al. Radiofrequency ablation vs endoscopic surveillance for patients with Barrett esophagus and low- grade dysplasia: a randomized clinical trial. JAMA 2014;311:1209-17.

13. Choi YNWH, Coremans G, Depeyper S, et al. Short term side effects after radiofrequency ablation. Are we ready to ablate non-dysplastic Barrett? Gut 2011;60(Suppl 3):A59.

14. Maccini M, Sehrt D, Pompeo A, et al. Biophysiologic considerations in cryoablation: a practical mechanistic molecular review. Int Braz J Urol 2011;37:693-6.

15. Erinjeri JP, Clark TW. Cryoablation: mechanism of action and devices. J Vasc Interv Radiol 2010;21(8 Suppl):S187-91.

16. Scholvinck DW, Kunzli HT, Kestens C, et al. Treatment of Barrett's esophagus with a novel focal cryoablation device: a safety and feasi- bility study. Endoscopy 2015;47:1106-12

17. Kunzli HT, Scholvinck DW, Meijer SL, et al. Efficacy of the cryoballoon focal ablation system for the eradication of dysplastic Barrett's esoph- agus islands. Endoscopy 2017;49:169-75

18. Sitaraman L. Use of the cryoballoon focal ablation system for the erad- ication of esophageal neoplasia: a single-center experience. Gastro- enterology 2016;150(4 Suppl 1):S266.

19. Truesdale CM, Soulen MC, Clark TW, et al. Percutaneous computed tomography-guided renal mass radiofrequency ablation versus cryoa- blation: doses of sedation medication used. J Vasc Interv Radiol 2013;24:347-50.

20. Johnston MH, Eastone JA, Horwhat JD, et al. Cryoablation of Barrett's esophagus: a pilot study. Gastrointest Endosc 2005;62:842-8.

21. Overwater A, Weusten B. Cryoablation in the management of Barrett's esophagus. Curr Opin Gastroenterol 2017;33:261-9.

22. Sharma P, Dent J, Armstrong D, et al. The development and validation of an endoscopic grading system for Barrett's esophagus: the Prague C \& M criteria. Gastroenterology 2006;131:1392-9. 
23. Friedland S, Triadafilopoulos G. A novel device for ablation of abnormal esophageal mucosa (with video). Gastrointest Endosc 2011;74:182-8.

24. van Vilsteren FG, Phoa KN, Alvarez Herrero L, et al. A simplified regimen for focal radiofrequency ablation of Barrett's mucosa: a ran- domized multicenter trial comparing two ablation regimens. Gastroint- est Endosc 2013;78:30-8.

25. Kunzli HT, Scholvinck DW, Phoa KN, et al. Simplified protocol for focal radiofrequency ablation using the HALO90 device: short-term efficacy and safety in patients with dysplastic Barrett's esophagus. Endoscopy 2015;47:592-7.

26. Dische S, Saunders M, Barrett A, et al. A randomised multicentre trial of CHART versus conventional radiotherapy in head and neck cancer. Radiother Oncol 1997;44:123-36.

27. Lara-Munoz C, De Leon SP, Feinstein AR, et al. Comparison of three rat- ing scales for measuring subjective phenomena in clinical research. I. Use of experimentally controlled auditory stimuli. Arch Med Res 2004;35:43-8.

28. Pouw RE, Gondrie JJ, Rygiel AM, et al. Properties of the neosquamous epithelium after radiofrequency ablation of Barrett's esophagus con- taining neoplasia. Am J Gastroenterol 2009;104:1366-73.

29. Shaheen NJ, Falk GW, lyer PG, et al. ACG clinical guideline: diagnosis and management of Barrett's esophagus. Am $J$ Gastroenterol 2016;111:30-50; quiz, 1.

30. Belghazi K, Pouw RE, Sondermeijer CM, et al. Safety and efficacy of circumferential radiofrequency ablation of Barrett's esophagus using a self-sizing RFA balloon catheter: results of a pilot study [abstract]. Gastrointest Endosc 2016;83:AB555.

31. van Vilsteren FG, Phoa KN, Alvarez Herrero L, et al. Circumferential balloon-based radiofrequency ablation of Barrett's esophagus with dysplasia can be simplified, yet efficacy maintained, by omitting the cleaning phase. Clin Gastroenterol Hepatol 2013;11:491-8

32. Phoa KN, Pouw RE, Bisschops R, et al. Multimodality endoscopic erad-ication for neoplastic Barrett oesophagus: results of a European multi- centre study (EURO-II). Gut 2016;65:555-62.

33. Canto MI, Abrams JA, Kunzli HT, et al. Nitrous oxide cryotherapy for treatment of esophageal squamous cell neoplasia: initial multicenter international experience with a novel portable cryoballoon ablation system (with video). Gastrointest Endosc 2018;87:574-81.

34. Allaf ME, Varkarakis IM, Bhayani SB, et al. Pain control requirements for percutaneous ablation of renal tumors: cryoablation versus radiofrequency ablationdinitial observations. Radiology 2005;237: 366-70.

35. Bastani H, Drca N, Insulander $\mathrm{P}$, et al. Cryothermal vs. radiofrequency ablation as atrial flutter therapy: a randomized comparison. Europace 2013;15:420-8.

36. Chen $\mathrm{YH}$, Lin $\mathrm{H}, \mathrm{Xie} \mathrm{CL}$, et al. Efficacy comparison between cryoablation and radiofrequency ablation for patients with cavotricuspid valve isthmus dependent atrial flutter: a meta-analysis. Sci Rep 2015;5:10910.

37. Deisenhofer I, Zrenner B, Yin YH, et al. Cryoablation versus radio- frequency energy for the ablation of atrioventricular nodal reen- trant tachycardia (the CYRANO Study): results from a large multicenter prospective randomized trial. Circulation 2010;122: 2239-45.

38. Farrar JT, Portenoy RK, Berlin JA, et al. Defining the clinically important difference in pain outcome measures. Pain 2000;88:287-94

39. Farrar JT, Young JP Jr, LaMoreaux L, et al. Clinical importance of changes in chronic pain intensity measured on an 11point numerical pain rating scale. Pain 2001;94:149-58.

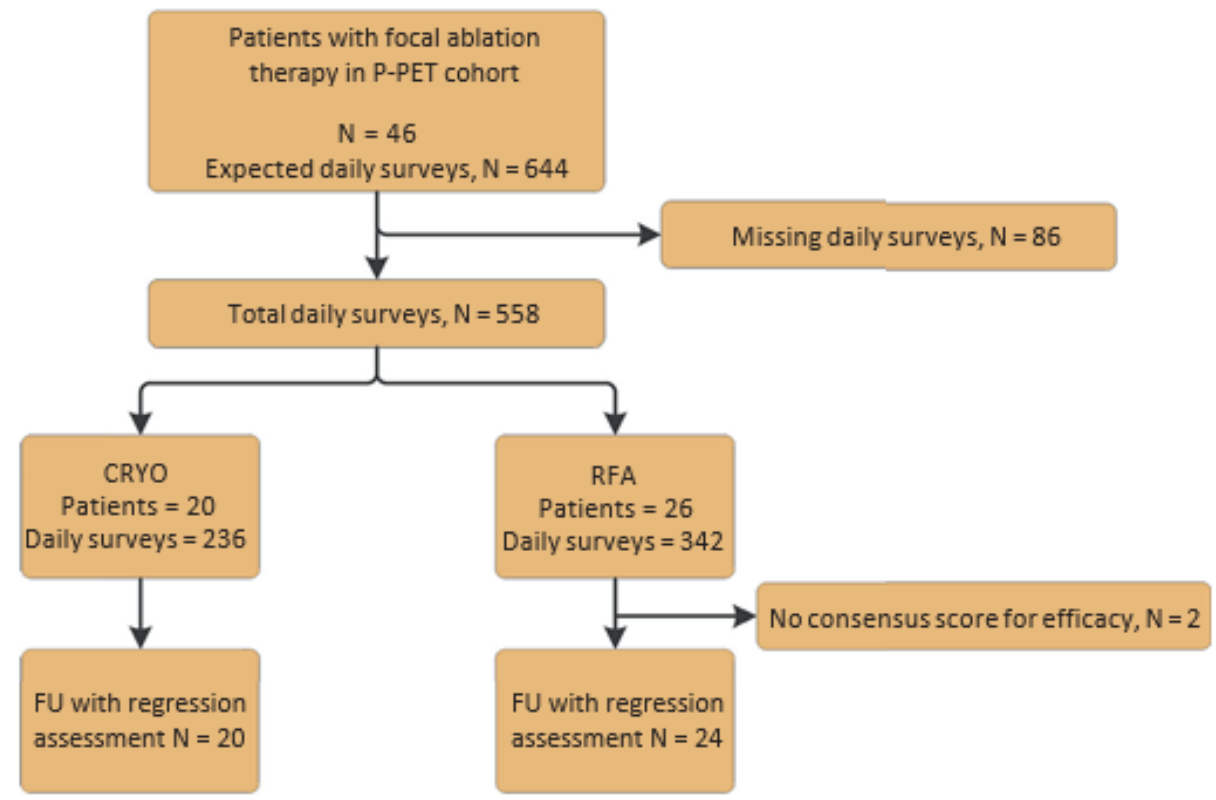

Figure 1. Flow chart for study patients. All patients with focal ablation therapy [CRYO or RFA] in the P-PET cohort were included in the study. All bu patients were eligible for the efficacy assessment. CRYO, Gryogblation; FU, follow-up; P-PET cohort, pQstprocedural pain after endoscopic therapy: Barrett's esophagus cohort; RFA, radiofrequency ablation. 


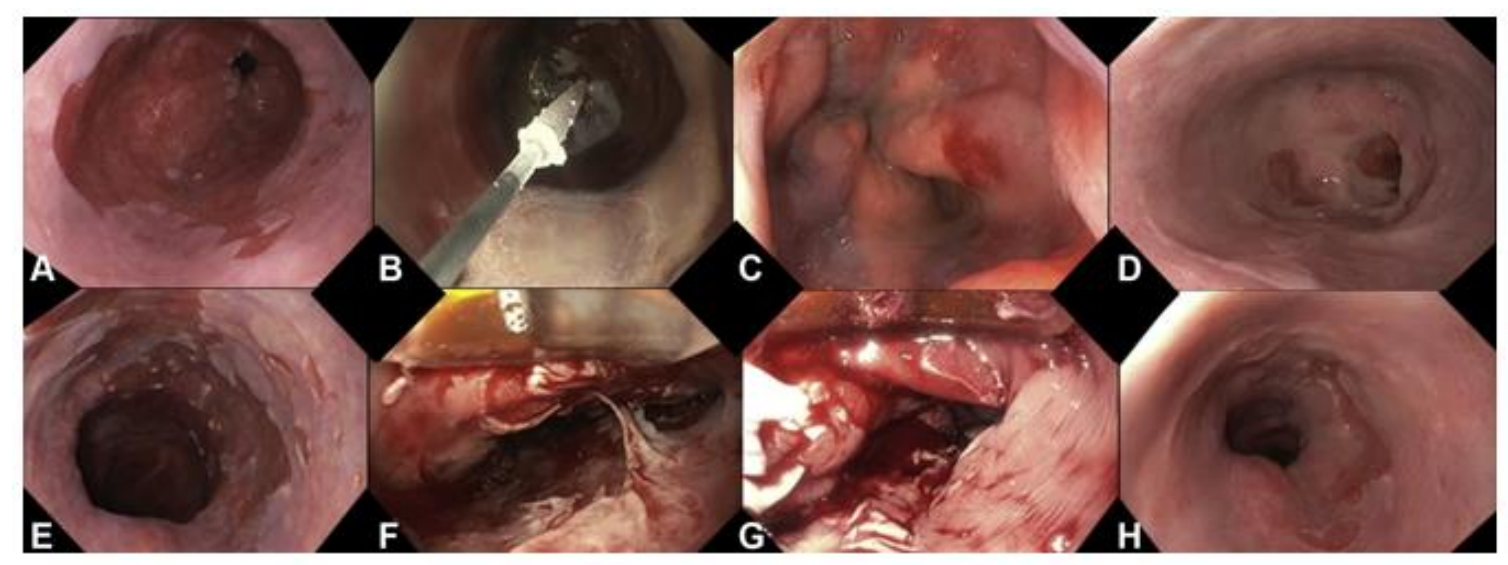

Figure 2. Baseline, treatment, and follow-up images for CRYO and RFA. The images show a typical case for CRYO [A-D) and RFA (E-H]. A, Initial BE before treatment. B, CbEAS treatment, ice patch visible at $60^{\prime}$ clock. C. SlbEAS effect directly post-treatment. D, Follow-up endoscopy 3 months after ClbEAS, with partial eradication of BE. E, Initial BE before treatment. F and G. Focal RFA treatment. Treatment effect is visible as sloughing mucosa with slight bleeding. H. Follow-up endoscopy 3 months after focal RFA with partial eradication of $B E$. CRYO, Grzoablation; $R F A$, radiofrequency ablation; $B E$, Barrett's esophagus; GbEAS, Grxoballoon focal ablation system.

\begin{tabular}{|c|c|c|c|}
\hline & CRYO group (n [ 20) & RFA group (n $\mathbf{\Gamma} 26$ ) & Pvalue \\
\hline Sex, male & $17(85)$ & $21(81)$ & .71 \\
\hline Median age, y (IQR) & $66(62-71)$ & $68(63-74)$ & .38 \\
\hline \multicolumn{4}{|l|}{ BE length before focal ablation, $\mathrm{cm}$} \\
\hline Circumferential extent, median (IQR) & $0(0-0)$ & $0(0-0)$ & .45 \\
\hline Maximum extent, median (IQR) & $2(1-3)$ & $1(0-3)$ & .25 \\
\hline Most proximal BE island, median (IQR) & $3(2-5)$ & $2(1-5)$ & .61 \\
\hline \multicolumn{4}{|c|}{ Worst histology diagnosis before first treatment } \\
\hline LGD & $9(45)$ & $14(54)$ & .59 \\
\hline HGD or $M E A C$ & $11(55)$ & $12(46)$ & \\
\hline \multicolumn{4}{|l|}{ Prior treatment } \\
\hline ER and ablation & $4(20)$ & $7(27)$ & .54 \\
\hline ER & $6(30)$ & $5(19)$ & \\
\hline Ablation & $2(20)$ & $9(35)$ & \\
\hline \multicolumn{4}{|l|}{ Type of sedation } \\
\hline Propofol & $19(95)$ & $22(85)$ & .26 \\
\hline Midazolam & $1(5)$ & $4(15)$ & \\
\hline
\end{tabular}

Values are $n$ (\%) unless otherwise defined. Baseline characteristics were not significantly different for the CRYO and the RFA group.

$B E$, Barrett's esophagus; $C R Y O$, sXeablation; $E R$, endascopic resection; $H G D$, high-grade dysplasia; lQR, interquartile range; LGD, low-grade dysplasia; $m E A G$, mucosal esophageal adenocarcinoma; RFA, radiofrequency ablation. 


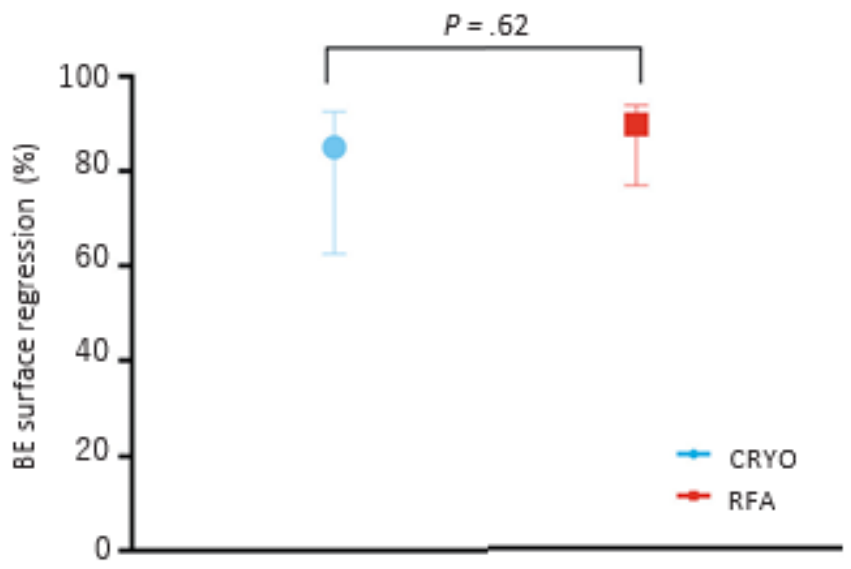

Figure 3. Efficacy. BE surface regression for both groups, scored by 2 independent $B E$ expert endoscopists at 3 months follow-up after a single treatment session. The blue circle (CRYO) and red square (RFA) indicate median scores and error bars denote interquartile ranges. $B E$, Barrett's esophagus; CRYO, crxoablatiin; RFA, radiofrequency ablation.

\begin{tabular}{lccc}
\hline TABLE 2. Post-treatment characteristics & & & \\
\hline & CRYO group (n [ 20) & RFA group (n [ 26) & P value \\
BE surface regression, median (IQR) & $88(63-94)$ & $90^{*}(77-94)$ & .62 \\
\hline Images per patient, median (IQR) & $9(7-11)$ & $9(8-10)$ & $2(8)$ \\
\hline Stenosis & $0(0)$ & .91 & $0(0)$ \\
\hline Other adverse events & $0(0)$ & NA \\
\hline
\end{tabular}

Values are $\mathrm{n}(\%)$ unless otherwise defined. Efficacy after a single treatment session was defined as the BE surface regression percentage and was independently assessed by 2 expert Soldescepists. This score was comparable for CRYO and RFA.

BE, Barrett's esophagus; CRYO, coloablatian; IQR interquartile range; RFA, radiofrequency ablation; $N A$, not applicable.

"BE surface regression was assessed for 24 patients. Two patients with poor image quality that hampered adequate assessment of the BE surface regression were excluded for this analysis. 

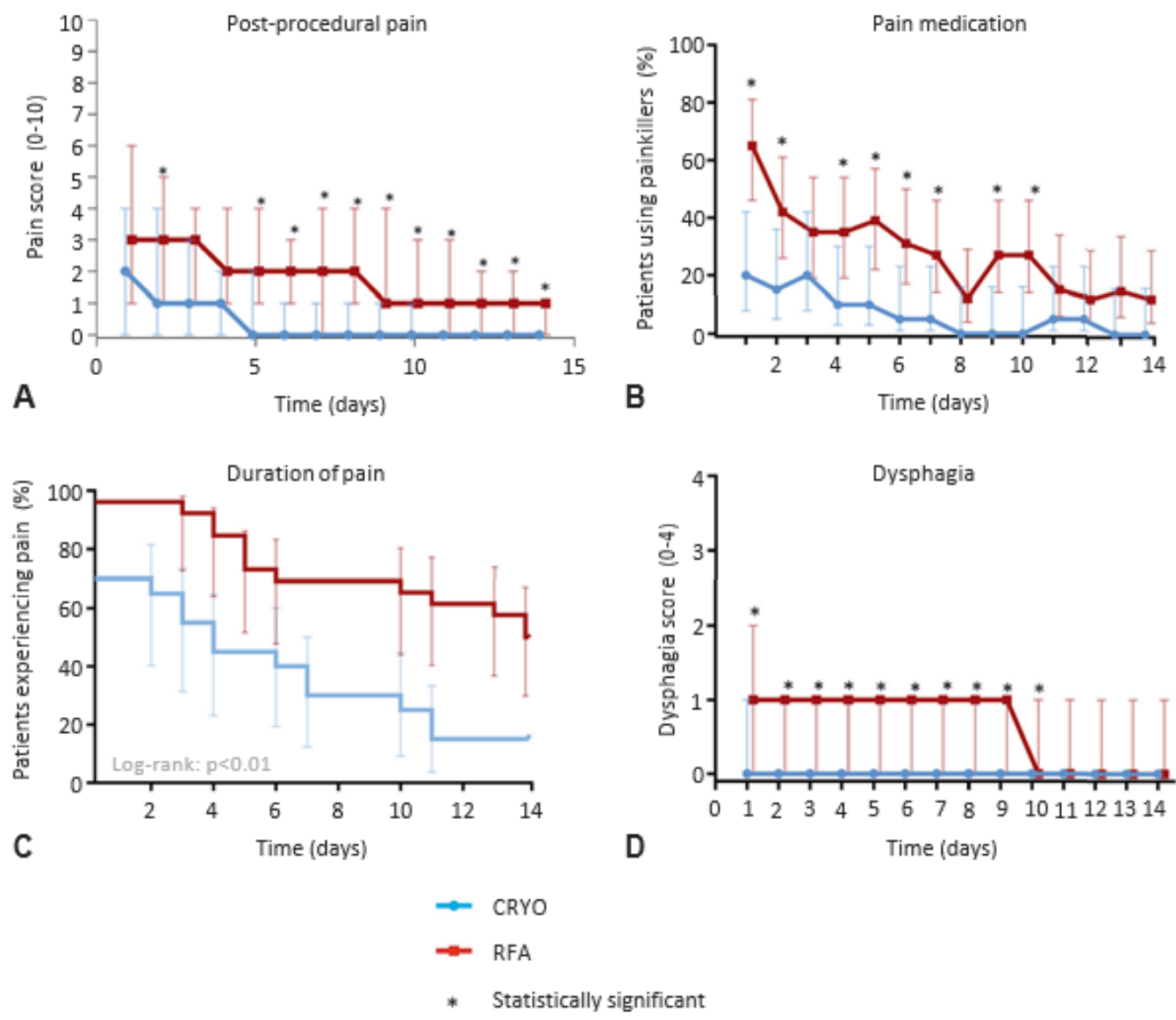

Figure 4. Postprocedural pain. A, Median daily pain scores through 14 days after treatment as recorded in the electronic diary. B, Percentage of patients using pain medication. C, Duration of pain, defined as number of days until a pain score of $O$. D, Median dysphagia scores through 14 days after treatment. Blue circles (CRYO) and red squares (RFA] indicate median scores and error bars denote interquartile ranges. CRYO, Crrsablation; $R F A$, radiofrequency ablation.

\begin{tabular}{|c|c|c|}
\hline & B coefficient $[95 \% \mathrm{Cl}]$ & $P$ value \\
\hline \multicolumn{3}{|c|}{ Unovariable analysis } \\
\hline Treatment & $-14.9[-26.7$ to -3.1$]$ & .02 \\
\hline \multicolumn{3}{|c|}{ Multivariable analysis } \\
\hline Treatment & $-17.3[-29.8$ to -4.7$]$ & $<.01$ \\
\hline Age & $-.6[-1.4$ to .2$]$ & .15 \\
\hline Sex & $-2.2[-19.3$ to 14.8$]$ & .80 \\
\hline BE length & $1.6[-2.0$ to 5.1$]$ & .37 \\
\hline Prior ablation & $-8.2[-20.8$ to 4.4$]$ & .19 \\
\hline Prior endoscopic resection & $2.8[-9.6$ to 15.2$]$ & .65 \\
\hline Sedation type & $2.7[-18.4$ to 23.8$]$ & .80 \\
\hline
\end{tabular}

On multivariable linear regression analysis correcting for potential confounders in the association between treatment type and cumulative pain, CRYO was associated with significantly less cumulative pain compared with RFA (decrease of 18.1 in AUC; $P<.01$ ). Treatment is presented as 0 for RFA and 1 for $C R Y O$; age in years, sex as 0 for female, BE length is in $\mathrm{cm}$, prior ablation and endoscopic resection as 0 for no, sedation type as 0 for propofol, and 1 for conscious sedation with midazolam. Example: prior ablation therapy is associated with a decrease of 13.8 in the area under the curve for cumulative pain ( $P$ Z .02)

$B E$, Barrett's esophagus; $C$, confidence interval; CRYO, scugballego ablation; RFA, radiafrequency ablation. 

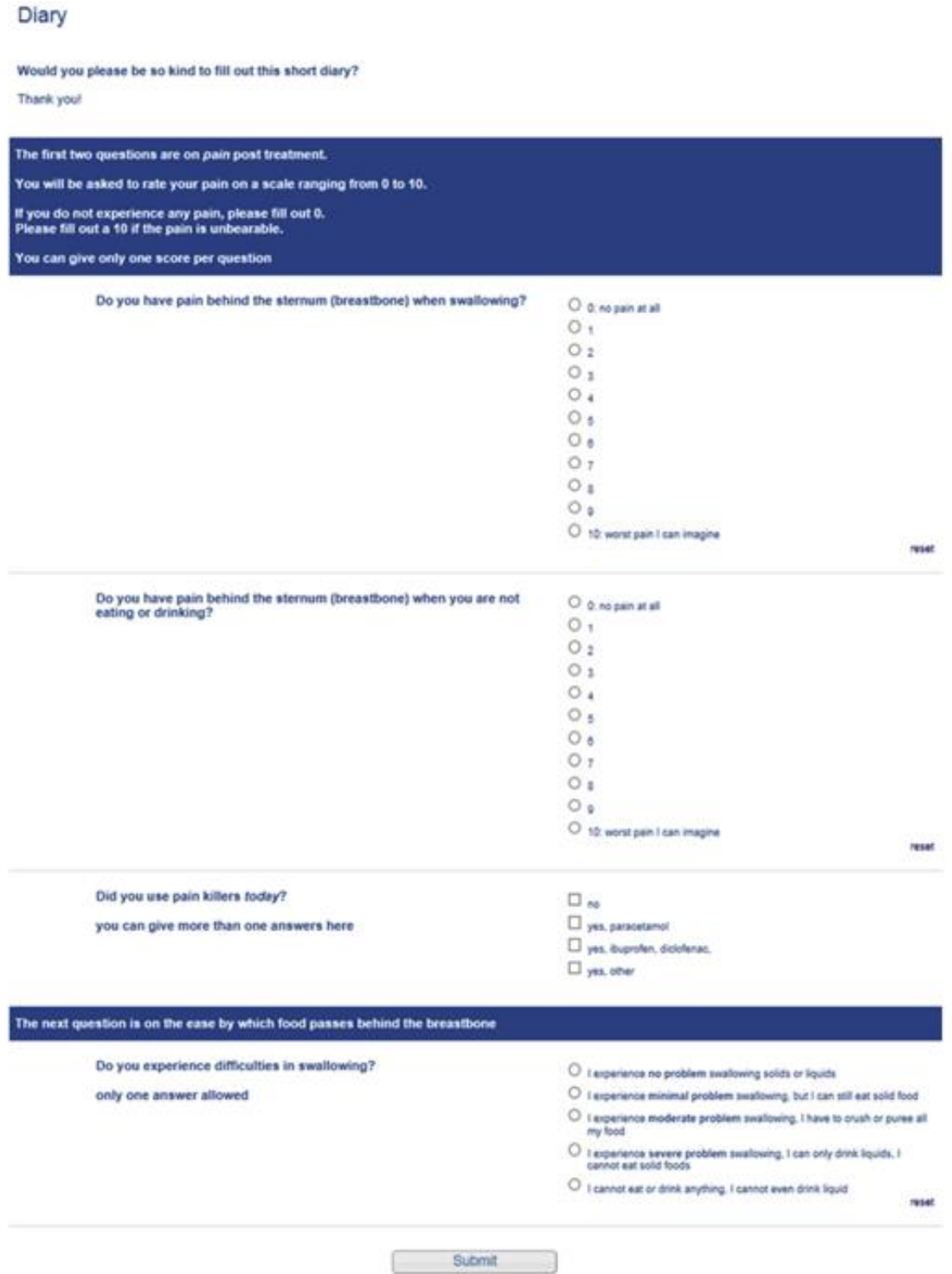

Supplementary Figure 1. Daily survey in the electronic diary. This survey questioning pain, dysphagia, and pain medication was sent out daily to all patients through 14 days after treatment. 